\title{
The Structure of He-Irradiated Gold Twist Boundary
}

\author{
S. Dey, ${ }^{*}$ and N. D. Browning $*, * *, * * *$
}

* Chemical Engineering and Materials Science, University of California, Davis, CA 95616

** Molecular and Cellular Biology Department, University of California, Davis, CA 95616

*** Condensed Matter and Materials Division, Physical and Life Sciences Directorate, Lawrence

Livermore National Laboratory, 7000 East Ave., Livermore, CA 94550-9234

Grain boundaries (GBs) in materials bring about interesting properties not seen in perfect crystals. Materials exhibit changes in atomic co-ordination, non-stoichiometry, and partially-occupied atomic columns at grain boundaries. Their presence provides an opportunity to tailor and improve material properties to our liking. One such example is the doping of perovskite GB to improve its superconducting behavior (critical current density) [1].

To understand how a GB affects the material's properties, its structure must be known. In each crystalline grain, atoms arrange themselves in a regular repetitive pattern but at GBs the regular arrangement of atoms gets disturbed. One special category of GB is the low angle boundary, which is comprised of networks of dislocations. Two types of simple low angle GBs are tilt boundaries, comprised of edge dislocations, and twist boundaries, comprised of screw dislocations. Gold twist boundaries have been studied by X-ray diffraction [2], high resolution electron microscopy [3], and theoretical calculation [4]. Theoretical calculation and Transmission Electron Microscopy (TEM) are used to complement each other in studies of these boundaries. By using TEM, it is possible to determine GB's structure, i.e., types of dislocation cores, distance between dislocation cores, Burgers vector, or the presence of foreign material. Here we investigate a Helium (He)-irradiated $\mathrm{Au}$ $1^{0}$ twist $\mathrm{GB}$ (Au as representative of a face-centered cubic crystal) by using the TEM and Scanning Transmission Electron Microscopy (STEM) equipped with a spherical aberration corrector. The bright-field and Z-contrast images will provide complementary information about the samples.

The sample is a bi-crystal with a twist GB. Au single crystals are produced by evaporation on two salt crystal templates. These two single crystals are joined at a specific misorientation, followed by removal of the substrates. The specimen was then ion implanted $\left({ }^{3} \mathrm{He}^{+}\right)$to a composition of 1.2-2.4 at. $\%$ at the GB.

Preliminary results are presented in Figures 1 and 2. Figures 1(a) and (b) show the presence of a square grid of screw dislocations at the boundary. Rectangular grids of screw dislocations are also reported at the $\mathrm{Au}$ twist GB in previous studies. GB dislocations having large Burgers vectors and localized cores produce long-range strain fields that are strong enough to be detected by diffraction contrast in TEM [5]. Figures 2(a) and (b) show an area at high magnification. Locations of He bubbles are circled. Further studies will explain the structure of these dislocation cores, distribution of He bubbles at the boundary and in the body of the crystal, and interaction of the He bubbles with GBs.

The specimen for this investigation was provided by Amit Misra and Michael Nastasi of the Center for Materials at Irradiation and Mechanical Extremes (CMIME) at the Los Alamos National Laboratory. 


\section{References}

[1] D. M. Feldmann et al., Nature, 414 (2001) 368.

[2] T. Y. Tan et al., Phi. Mag., 27 (1973) 1225.

[3] T. Y. Tan et al., Phi. Mag., 43 (1975) 575.

[4] I. Majid et al., Physical Review B, 40 (1989) 2779.

[5] T. Schober et al., Phys. Stat. Sol. (a), 6 (1971) 103.

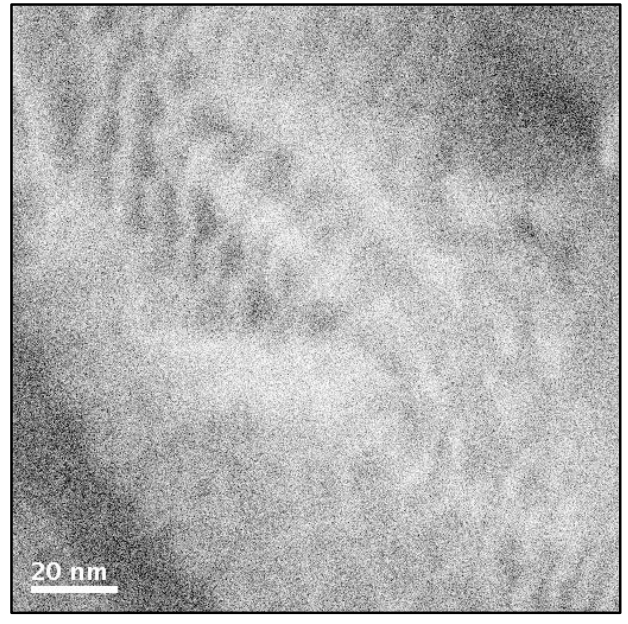

(a)

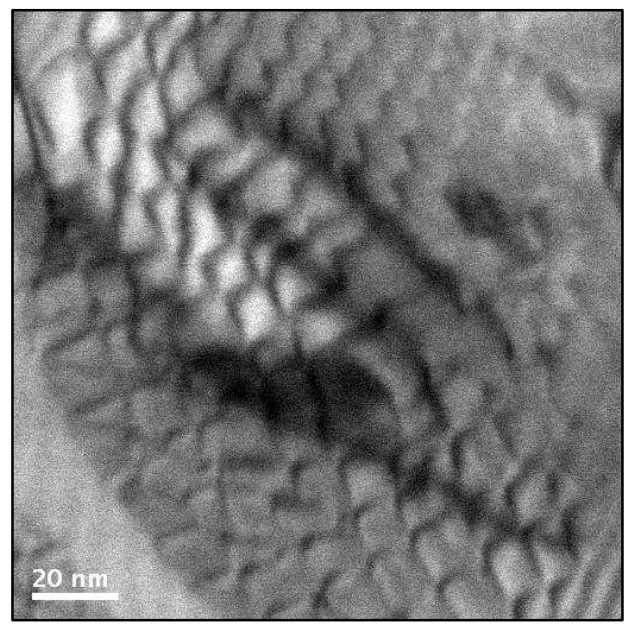

(b)

FIG. 1. STEM (a) DF image (b) BF image of Au Twist Boundary showing square grid of screw dislocations.

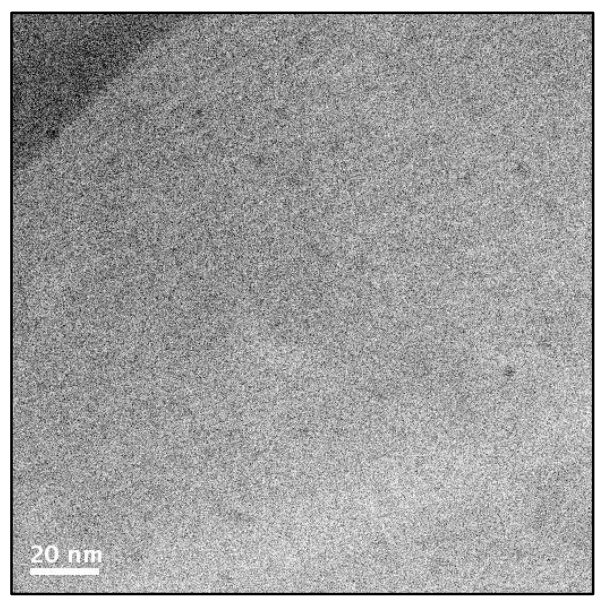

(a)

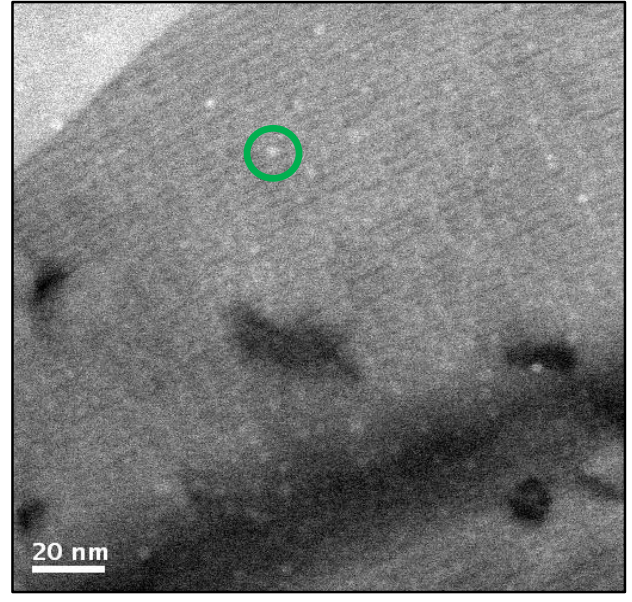

(b)

FIG. 2. STEM image showing He bubbles (circled on image b) - (a) DF , (b) BF. 